\title{
Imaging and Spectroscopy with the James Webb Space Telescope
}

\author{
George Sonneborn \\ Laboratory for Observational Cosmology, Code 665 \\ NASA Goddard Space Flight Center \\ Greenbelt, MD 20771, USA \\ email: george.sonneborn@nasa.gov
}

\begin{abstract}
The James Webb Space Telescope (JWST) is a large, infrared-optimized space telescope scheduled for launch in 2013. JWST will find the first stars and galaxies that formed in the early universe, connecting the Big Bang to our own Milky Way galaxy. JWST will peer through dusty clouds to see stars forming planetary systems, connecting the Milky Way to our own Solar System. JWST's instruments are designed to work primarily in the infrared range of $1-28 \mu \mathrm{m}$, with some capability in the visible range. JWST will have a large segmented mirror, $\sim 6.5 \mathrm{~m}$ in diameter, and will be diffraction-limited at $2 \mu \mathrm{m}(<0.1$ arcsec resolution). JWST will be placed in an L2 orbit about 1.5 million $\mathrm{km}$ from the Earth. The instruments will provide imaging, coronography, and multi-object and integral-field spectroscopy across the $1-28 \mu \mathrm{m}$ wavelength range. The breakthrough capabilities of JWST will enable new studies of massive stars from the Milky Way to the early universe.
\end{abstract}

Keywords. telescopes - space vehicles: instruments - instrumentation: high angular resolution - instrumentation: spectrographs

\section{Mission Overview}

The James Webb Space Telescope (JWST) is a large $(6.5 \mathrm{~m})$, cold $(T \sim 40 \mathrm{~K})$, infraredoptimized space observatory being built for launch in 2013 on an Ariane 5 ESA rocket into orbit around the Sun-Earth Lagrange point L2. The observatory will have four instruments: a camera, a multi-object spectrograph, and a tunable filter imager will cover the near-infrared spectrum $(0.6<\lambda<5.0 \mu \mathrm{m})$. A mid-infrared instrument will provide imaging and spectroscopy from $5.0<\lambda<28 \mu \mathrm{m}$. Coronography will also be possible across the entire JWST bandpass. JWST is a cooperative project between NASA and the European and Canadian Space Agencies (ESA and CSA). The mission design and development is led and managed by NASA's Goddard Space Flight Center. JWST will be operated for NASA and the scientific community by the Space Telescope SCience Institute.

The JWST science goals, observatory design, operational concept, and expected performance are described in detail by Gardner et al. (2006, see the link on the JWST web site www.jwst.nasa.gov/science.html) to download the reprint of this large paper). The present contribution is a highly abbreviated version of the information contained in Gardner et al.

The JWST science goals are divided into four themes. The End of the Dark Ages: First Light and Reionization theme is to identify the first luminous sources to form and to determine the ionization history of the early universe. The Assembly of Galaxies theme is to determine how galaxies and the dark matter, gas, stars, metals, morphological structures, and active nuclei within them evolved from the epoch of reionization to the present day. The Birth of Stars and Protoplanetary Systems theme is to unravel the 
birth and early evolution of stars, from infall on to dust-enshrouded protostars to the genesis of planetary systems. The Planetary Systems and the Origins of Life theme is to determine the physical and chemical properties of planetary systems, including our own, and investigate the potential for the origins of life in those systems.

To enable these observations, JWST consists of a telescope, an instrument package, a spacecraft and a sunshield. The instrument package contains the four science instruments (SIs) and a fine guidance sensor. The Sun, Earth, and Moon are always behind the sunshield, so that the telescope and instruments are passively cooled to $T \sim 40 \mathrm{~K}$. The spacecraft, located on the sunward side of the sunshield, provides pointing, power, orbit maintenance and communications. The JWST operations plan is based on that used for previous space observatories, and the majority of JWST observing time will be allocated to the international astronomical community through annual peer-reviewed proposal opportunities.

The telescope is a deployable optical system consisting of 18 hexagonal beryllium segments that provide diffraction-limited performance at $2 \mu \mathrm{m}$. The mirror segments are adjustable in orbit in six degrees of freedom. Image quality is maintained by periodic monitoring of the wavefront errors by NIRCam (Section 2.1) and biweekly updates to the mirror positions. The wavelength range of JWST and the SIs spans 0.6 to $28 \mu \mathrm{m}$, limited at the short end by the gold coatings on the primary mirror and at the long end by the detector technology.

JWST is designed to provide instantaneous sky coverage over the solar elongation range of $85^{\circ}$ to $135^{\circ}$ (95 $95^{\circ}$ to $45^{\circ}$ from the anti-solar direction). There s a continuous viewing zone within $5^{\circ}$ of both the north and south ecliptic poles. Thirty percent of the sky can be viewed continuously for at least 197 continuous days. All regions of the sky have at least 51 days of continuous visibility per year. The JWST architecture provides an instantaneous visibility of $\sim 40 \%$ of the sky.

The sunshield attenuates the incident solar radiation by a factor of $\sim 10^{5}$. This solar attenuation is a result of the five-layer configuration of the sunshield. Its physical size and shape determine the instantaneous sky coverage for the observatory. By reducing the solar radiation to the milliwatt level, the observatory has an intrinsically stable point spread function at all solar orientations within the allowed range.

Many JWST observations will be background limited. The background is a combination of in-field zodiacal light, scattered thermal emission from the sunshield and telescope, scattered starlight, and scattered zodiacal light. Over most of the sky, the zodiacal light dominates at wavelengths $\lambda<10 \mu \mathrm{m}$. The sensitivities of the JWST instruments are given in Table 1.

\section{JWST Science Instruments}

JWST has four science instruments: NIRCam, NIRSpec, TFI, and MIRI. A cryo-cooler will be used for cooling the MIRI focal plane and its Si:As detectors. The near-infrared detector arrays in the other three instruments are passively cooled $\mathrm{HgCdTe}$ of HAWAII II heritage. The instrument module also houses the Fine Guidance Sensor (FGS, provided by CSA) and a computer that directs the daily science observations based on plans received from the ground. The science instruments and FGS have non-overlapping FOVs. Simultaneous operation of all science instruments is possible. This capability will be used for parallel calibration, including darks and possibly sky flats. FGS is used for guide star acquisition and fine pointing. Its FOV and sensitivity are sufficient to provide a greater than $95 \%$ probability of acquiring a guide star for any valid pointing direction and roll angle. 
Table 1. JWST Instrument Sensitivities

\begin{tabular}{llll}
\hline Instrument/Mode & $\lambda(\mu \mathrm{m})$ & Bandwidth & Sensitivity \\
\hline NIRCam & 1.1 & $\mathrm{R}=4$ & $12.1 \mathrm{nJy}, \mathrm{AB}=28.7$ \\
NIRCam & 2.0 & $\mathrm{R}=4$ & $10.4 \mathrm{nJy}, \mathrm{AB}=28.9$ \\
TFI & 3.5 & $\mathrm{R}=100$ & $126 \mathrm{nJy}, \mathrm{AB}=26.1$ \\
NIRSpec/Low Res & 3.0 & $\mathrm{R}=100$ & $120 \mathrm{nJy}, \mathrm{AB}=26.2$ \\
NIRspec/Med Res & 2.0 & $\mathrm{R}=1000$ & $1.64 \times 10^{-18} \mathrm{erg} \mathrm{s}^{-1} \mathrm{~cm}^{-2}$ \\
MIRI/Broad-Band & 10.0 & $\mathrm{R}=5$ & $700 \mathrm{nJy}, \mathrm{AB}=24.3$ \\
MIRI/Broad-Band & 21.0 & $\mathrm{R}=4.2$ & $7.3 \mu \mathrm{Jy}, \mathrm{AB}=21.7$ \\
MIRI/Spect. & 9.2 & $\mathrm{R}=2400$ & $1.0 \times 10^{-17} \mathrm{erg} \mathrm{s}^{-1} \mathrm{~cm}^{-2}$ \\
MIRI/Spect. & 22.5 & $\mathrm{R}=1200$ & $5.6 \times 10^{-17} \mathrm{erg} \mathrm{s}^{-1} \mathrm{~cm}^{-2}$ \\
\hline
\end{tabular}

NOTE - Sensitivity is evaluated for a point source detected at $10 \sigma$ in $10000 \mathrm{~s}$.

\subsection{Near-Infrared Camera (NIRCam)}

NIRCam provides filter imaging and coronography in the 0.6 to $5.0 \mu \mathrm{m}$ range with wavelength multiplexing. It includes the ability to sense the wavefront errors of the observatory. NIRCam uses a dichroic to simultaneously observe the short (0.6 to $2.3 \mu \mathrm{m})$ and long $(2.4$ to $5.0 \mu \mathrm{m}$.) wavelength light paths from the same field of view.

The instrument contains a total of ten $2 \mathrm{~K} \times 2 \mathrm{~K}$ detectors, including those in the identical redundant optical trains. The short wavelength arm in each optical train contains four detectors,optimized for the $0.6-2.3 \mu \mathrm{m}$ wavelength range, with a small gap $(\sim 3 \mathrm{~mm}$ $=\sim 5$ arcsec) between adjacent detectors. The detectors will all have thinned substrates to avoid cosmic ray scintillation issues, as well as to extend their sensitivity below 0.85 $\mu \mathrm{m}$. Each optical train contains filter and pupil wheels that hold a range of filters and wavefront sensing optics.

To enable the coronagraphic imaging, each of the two identical optical trains in the instrument contains a traditional focal plane coronagraphic mask plate held at a fixed distance from the detectors. This mounting ensures that the coronagraph spots are always in focus at the detector plane. Each coronagraphic plate is transmissive, and contains a series of spots of different sizes, including linear and radia-sinc occulters, to block the light from a bright object.

\subsection{Near-Infrared Spectrograph (NIRSpec)}

NIRSpec is a near infrared multi-object dispersive spectrograph provided by ESA that is capable of simultaneously observing up to $\sim 100$ sources over a field-of-view (FOV) larger than $3 \times 3$ arcmin. In addition to the multi-object capability, it includes fixed slits and an integral field unit (IFU) for imaging spectroscopy. Six gratings will yield resolving powers of $\mathrm{R} \sim 1000$ and $\sim 2700$ in three spectral bands, spanning the range 1.0 to 5.0 $\mu \mathrm{m}$. A prism will yield $\mathrm{R} \sim 100$ over 0.6 to $5.0 \mu \mathrm{m}$.

Targets in the FOV are normally selected by opening groups of shutters in a microshutter assembly to form multiple apertures. The micro-shutter assembly itself consists of a mosaic of 4 subunits producing a final array of approximately 750 (spectral) by 350 (spatial) individually addressable shutters with $200 \times 450$ milliarcsec (mas) openings and $250 \times 500$ mas pitch. The minimum aperture size is 1 shutter (spectral) by 1 shutter (spatial) at all wavelengths. Multiple pointings may be required to avoid placing targets near the edge of a shutter and to observe targets with spectra that would overlap if observed simultaneously at the requested roll angle. The nominal slit height perpendicular to the dispersion direction is three shutters in all wavebands. These provide background spectra adjacent to the science target in the central shutter. In the open configuration, a shutter 
passes light from the fore-optics to the collimator. A slitless mode can be configured by opening all of the micro shutters. As the shutters are individually addressable, long slits, diagonal slits, Hadamard transform masks, and other patterns can also be configured.

In addition to the slits defined by the micro-shutter assembly, NIRSpec also includes five fixed slits that can be used for high-contrast spectroscopy. They are placed in a central strip of the aperture focal plane between sub-units of the micro-shutter assembly. Three fixed slits are 3.5 arcsec long and 200 mas wide. One fixed slit is 4 arcsec long and 400 mas wide for increased throughput at the expense of spectral resolution. One fixed slit is 2 arcsec long and 100 mas wide for brighter targets.

\subsection{Tunable Filter Imager (TFI)}

The TFI, built by CSA, provides narrow-band near-infrared imaging over a field of view of $2.2 \times 2.2 \operatorname{arcmin}^{2}$ with a spectral resolution $\mathrm{R} \sim 100$. The etalon design allows observations at wavelengths of $1.6 \mu \mathrm{m}$ to $2.6 \mu \mathrm{m}$ and $3.1 \mu \mathrm{m}$ to $4.9 \mu \mathrm{m}$. The gap in wavelength coverage allows the single channel to reach more than one octave in wavelength.

The TFI incorporates four coronagraphic occulting spots permanently to one side of the field of view, and occupying a region 20 by 80 arcsec. A set of selectable apodization masks is located at the internal pupil images of each channel by the filter wheels. The coronagraph is designed to deliver a contrast ratio of $\sim 10^{4}(10 \sigma)$ at 1 arcsec separation. The sensitivity is limited by speckle noise. Contrast ratios of $10^{5}$ may be achievable at sub-arcsec scales using roll or spectral deconvolution techniques.

\subsection{Mid-Infrared Instrument (MIRI)}

MIRI, provided by a consortium of European and U.S. institutions, is designed to obtain imaging, coronography, and spectroscopic measurements over the wavelength range 5 to $28 \mu \mathrm{m}$. A cryo-cooler will keep the MIRI Si:As detectors at $T \sim 6 \mathrm{~K}$. The optical bench contains two actively cooled subcomponents, an imager and IFU spectrograph, plus an on-board calibration unit. The imager module provides broad-band imaging, coronography, and low-resolution $(\mathrm{R} \sim 100,5-10 \mu \mathrm{m})$ slit spectroscopy using a single $1024 \times 1024$ pixel Raytheon Si:As detector with $25 \mu \mathrm{m}$ pixels. The coronagraphic masks include three phase masks for a quadrant-phase coronagraph and one opaque spot for a Lyot coronagraph. The coronagraphic masks each have a square field of view of $26 \times 26$ arcsec and are optimized for particular wavelengths.

The IFU obtains simultaneous spectral and spatial data on a small region of sky. The spectrograph field of view is next to that of the imager so that accurate positioning of targets will be possible by locating the image with the imager channel and off-setting to the spectrograph. The light is divided into four spectral ranges by dichroics, and two of these ranges are imaged onto each of two detector arrays. A full spectrum is obtained by taking exposures at each of three settings of the grating wheel. The spectrograph uses four image slicers to produce dispersed images of the sky on two $1024 \times 1024$ detectors, providing $\mathrm{R} \sim 3000$ integral field spectroscopy over the 5 to $29 \mu \mathrm{m}$ wavelength range, although the sensitivity of the detectors drops longward of $28 \mu \mathrm{m}$.

\section{References}

Gardner, J. P., Mather, J. C., Clampin, M., et al. 2006, Sp. Sci. Rev., 123, 485 